Meta

Journal des tradlucteurs

Translators' Journal

\title{
Étude terminologique des scies manuelles (III)
}

\section{Marie Proulx}

Volume 29, numéro 2, juin 1984

URI : https://id.erudit.org/iderudit/003875ar

DOI : https://doi.org/10.7202/003875ar

Aller au sommaire du numéro

Éditeur(s)

Les Presses de l'Université de Montréal

\section{ISSN}

0026-0452 (imprimé)

1492-1421 (numérique)

Découvrir la revue

Citer ce document

Proulx, M. (1984). Étude terminologique des scies manuelles (III). Meta, 29(2),

182-188. https://doi.org/10.7202/003875ar d'utilisation que vous pouvez consulter en ligne.

https://apropos.erudit.org/fr/usagers/politique-dutilisation/ 


\section{ÉTUDE TERMINOLOGIQUE DES SCIES MANUELLES (III)}

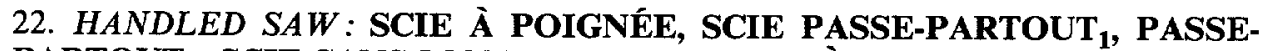 PARTOUT, ${ }_{1}$ SCIE SANS MONTURE (rare), SCIE À POIGNET (rare).}

Scie dont la lame est libre et munie d'une ou de deux poignées.

La scie à poignée est une scie dont la rigidité de la lame provient, non pas de la tension obtenue grâce à une monture, mais bien de la tension interne de la lame, qui est épaisse et généralement assez large, et ne se déforme pas pendant le travail. L'ouvrier l'utilise pour les opérations où une monture le gênerait. La famille des scies à poignée, dont le groupe le plus important est celui des scies à main, ou égoïnes, comprend des scies de dimensions très variées, qui vont de la petite scie passe-partout (env. $20 \mathrm{~cm}$ ) à la scie de long (env. $2 \mathrm{~m}$ ).

Les scies passe-partout sont munies d'une ou de deux poignées, selon le cas, qui peuvent être fixes ou amovibles, sous forme de cylindres de bois, de poignées droites (straight handle), fermées (de type " égoïne ") (closed handle), ou ouvertes (de type " pistolet ") (open handle).

Liste des scies à poignée

open pit saw (broad)

open pit saw (narrow)

(two-man) crosscut saw

one-man crosscut saw

hand saw

(crosscut) hand saw

rip saw

grafting saw

panel saw

compass saw

keyhole saw

pruning saw

flooring saw

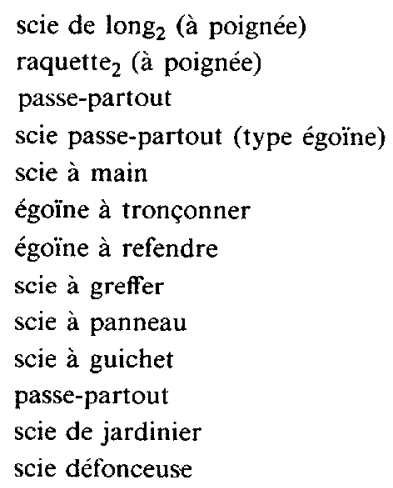

Remarque 1. "Passe-partout" est un terme générique que "scie à poignée" a aujourd'hui remplacé ; cependant, il n'est pas sorti de l'usage, puisqu'il désigne encore deux scies de cette famille, la grande scie passe-partout (voir TWO-MAN CROSS CUT $S A W$ ) et la petite (voir KEYHOLE SAW). 
Remarque 2. E $\mathrm{I}_{i}$ Europe, les ouvriers préfêrent encore utiliser les scies à monture, plus traditionnelles, et ne se servent que de quelques-unes des nombreuses scies à poignée, tandis que en Angleterre et en Amérique les scies à monture sont presque inconnues; ce sont les scies à poignée qui ont été popularisées, et c'est plutôt sur celles-ci que s'est arrêtée l'énergie des fabricants d'outils.

23. BROAD OPEN PIT SAW, PIT SAW $W_{3}$ PIT-SA $W_{3}$, OPEN PIT SAW, LONG SAW (1846) (arch.), WHIP SA $W_{1}$ (1677) (arch.), WHIPSA W (arch.) : SCIE DE LONG

Scie à poignée, de très grande dimension, réservée au sciage droit de débit premier dans le sens des fibres du bois.

Cette scie est une version " moderne" de la scie de long montée traditionnelle. Ce modèle, où le châssis est supprimé, est apparu en Angleterre vers la deuxième moitié du XVIII' siècle (où il a remplacé d'ailleurs immédiatement toutes les scies de long montées); on l'utilisait également dans quelques localités de Bretagne et de Normandie.

La lame de cette scie de long diffère de celle de la scie montée en ce qu'elle est plus épaisse, plus large (env. $10 \mathrm{~cm}$ à une extrémité et 25 à l'autre) et faite d'acier rigide. L'extrémité la plus large de la lame est munie d'une poignée perpendiculaire au plan de la lame, appelée «fer aux planches (tiller), qui consiste en un cylindre de bois passé dans la douille d'un bras en fer rivé à la lame ; l'autre extrémité peut être munie d'une poignée fixe du même type, ou amovible, appelée "menotte" (box).

Cette scie porte beaucoup de voie : ses dents dites " en gorge " gullet teeth; briar teeth sont espacées et profondes, crochues et inclinées, et ne travaillent que dans un sens, soit lorsqu'on tire la scie vers le bas (pushing saw ; down stroke saw).

La scie de long atteint environ deux mètres de longueur; c'est une scie de scieur utilisée pour l'équarrissage des grumes abattues au passe-partout et le débitage de cellesci en planches brutes.

Remarque 1. Le terme " whip saw ", de l'allemand "wippen ", illustre le mouvement de val-et-vient de la scie.

Remarque 2. "Broad open pit saw " est la forme étoffée ; " pit saw " est le terme le plus courant.

24. NARROW OPEN PIT SAW, PIT-SA $W_{4}$, PIT SA $W_{4}$, OPEN PIT SA $W_{2}$, TURNING $\mathrm{SA}_{2}$ (arch.), PIT TURNING SAW (1846) (arch.), FELLOE SAW (1846) (arch.) : RAQUETTE $_{2}$, SCIE RAQUETTE

Scie à poignée, de très grande dimension, réservée au sciage cintré de débit premier dans le sens des fibres du bois.

Cette scie est la version "à poignée " de la scie raquette, c'est-à-dire que le châssis y est supprimé. La lame de cette raquette est donc plus épaisse, plus large $(5 \mathrm{~cm}$ à une extrémité, $10 \mathrm{~cm}$ à l'autre) et faite d'acier plus rigide.

De même que la scie de long à poignée, elle est munie à son extrémité la plus large d'une poignée perpendiculaire au plan de la lame, le "fer aux planches ", et à l'autre d'une poignée fixe ou amovible, la "menotte".

C'est une scie de scieur qui atteint presque deux mètres et on l'utilise pour le débit de pièces légèrement cintrées (poutres de navires, p. ex.).

25. TWO-MAN CROSS CUT SAW, TWO-MAN CROSS-CUT SAW, CROSS-CUT SAW, CROSS-CUTTING SAW (1846), LUMBERMAN'S SAW, WHIP SAW (1677) (arch.), THWART SAW (arch.) : PASSE-PARTOUT (F.), SCIE PASSE-PARTOUT 
(F.), GODENDARD (CAN.), GODENDART (CAN.), SCIE PASSE-PARTOUT À DEUX MAINS (F.), PASSE PAR TOUT (1694) (arch.), PASSEPARTOUT (1690) (arch.).

Scie à poignée, de très grande dimension, réservée au sciage droit de débit premier à travers les fibres du bois.

La scie passe-partout, ou godendard, est une lame large (env. $15 \mathrm{~cm}$ ), généralement ventrée (bellied, breasted blade), mais parfois droite ou convexe, selon la tradition locale. Les poignées sont deux manches cylindriques en bois fixés à chacune des extrémités de la lame dans deux douilles rivées à celle-ci. La scie est ainsi manoeuvrée par deux hommes qui la tirent alternativement.

La lame est le plus souvent munie d'une denture rabotante épanouie (grauped teeth), dite "américaine " (American teeth), caractérisée par l'alternance de dents coupantes (cutting teeth) et rabotantes (naker teeth), ce qui permet de détacher, dans les deux sens du mouvement, des petits copeaux, au lieu de broyer le bois en sciure fine (on distingue plusieurs variantes de ce type de denture).

La scie passe-partout est une scie de bûcheron utilisée pour abattre les arbres et tronçonner les grumes en billes.

Remarque 1. "Two-man cross cut saw " est la forme étoffée ; " crosscut saw " est l'appellation la plus courante.

Remarque 2. La " lumberman's saw " est en fait une scie passe-partout à denture rabotante particulière, dite "lumberman".

Remarque 3. Les variantes orthographiques suivantes de "godendard" ont également été repérés : galendart, gadendart, guédendart, calendart, galondor.

26. ONE-MAN CROSSCUT SAW, ONE-MAN CROSS CUT SAW, ONE-MAN CROSS-CUT SAW : SCIE PASSE-PARTOUT TYPE ÉGOÏNE (F.), SCIEEGOÏNE (CAN.), GODENDARD POUR UN SEUL HOMME (CAN.) (rare), ARPON (CAN.) (rare), SCIE HARPON (CAN.) (rare), ZAG (arch.).

Scie à poignée, de très grande dimension, réservée au sciage droit de débit premier à travers les fibres du bois.

La scie passe-partout type égoïne ressemble à la scie passe-partout ; elle est cependant de dimension moindre ( $(1 \mathrm{a} 2 \mathrm{~m})$ et n'est munie que d'une seule poignée à son extrémité la plus large ; un petit montant amovible (removable handle) est parfois fixé sur le dos de la lame (montage par étrier) (stirrup joint), immédiatement devant la poignée égoïne, et sert de poignée auxiliaire à l'ouvrier ; ce montant peut être également posé à l'autre extrémité de la lame pour permettre à deux hommes de manier la scie. La scie égoïne est une scie de bûcheron qu'on utilise surtout pour le tronçonnage des grumes en billes.

27. HANDSAW, HAND SAW,$H A N D-S A W_{1}$ : EGOÏNE , EGOHINE (1690), SCIE À MAIN, SCIE EGGOHIN, SCIE EGOÏNE, SCIE HÉGOÏNE (rare), FEUILLET À POING (arch.).

Scie à poignée unique, fixée à l'extrémité la plus large de la lame, et qui n'est manoeuvrée que par un seul homme.

Les scies à main comptent pour la presque totalité de la famille des scies à poignées, et font partie de l'" outillage commun " aux artisans du bois. Leur lame est large, épaisse, pour ne pas être déformée pendant le travail, et flexible, du fait qu'elle n'est pas 
maintenue à ses deux extrémités ; elle est munie d'une poignée, rivée à son extrémité la plus large, le talon (butt ; heel ; handle end), et parfois d'un guidon (nib), à son extrémité étroite, ce qui sert à indiquer la fin de la course de l'outil. Elle peut avoir le dos droit (straight back saw) ou arqué (skew back saw), et sa rive dentée (cutting edge) est parfois légèrement convexe. La denture en est très fine et pointée vers le bout de la scie, la pointe (toe end ; front end ; point) : elle coupe donc lorsqu'on la pousse.

L'égoïne est d'une grande précision et elle produit un étroit trait de scie; elle se manoeuvre d'une seule main. On l'utilise pour diverses opérations, allant du débit second à la finition, exécutées sur des pièces larges qui ne peuvent être coupées par une scie montée dont le châssis gênerait le travail.

Les dimensions des égoïnes sont très variables (allant de 25 à $80 \mathrm{~cm}$ ) et diffèrent mème d'un corps de métier à un autre : ainsi, par exemple, les charpentiers utilisent une égoïne assez longue $(60$ à $80 \mathrm{~cm}$ ) et les menuisiers une plus courte $(45$ à $60 \mathrm{~cm})$. La scie à main est une scie très répandue en Angleterre et aux États-Unis (la poignée fermée, d'origine anglaise, est fermement établie dès le milieu du XVıI' siècle) et plusieurs modèles, qui diffèrent surtout par la longueur de la lame et le travail auquel on les destine, font partie de cette famille :

$\begin{array}{ll}\begin{array}{l}\text { rip saw }(70-75 \mathrm{~cm}) \\ \text { half rip saw }(65-70 \mathrm{~cm}) \\ \text { hand (crosscut) } \\ \text { broken space }(56-65 \mathrm{~cm}) \\ \text { (fine hand saw) }\end{array} & \text { égoïne à refendre de débit } \\ \text { panel saw }(25-50 \mathrm{~cm}) & \\ \text { fine panel }(25-50 \mathrm{~cm}) & \text { scie à panneau } \\ \text { plumber's saw }(25-45 \mathrm{~cm}) & \\ \text { siding saw }(25-50 \mathrm{~cm}) & \\ \text { chest saw }(25-40 \mathrm{~cm}) & \\ \text { tabile saw }(46-65 \mathrm{~cm}) & \\ \begin{array}{l}\text { compass saw }(20-46 \mathrm{~cm}) \\ \text { keyhole saw }(15-30 \mathrm{~cm})\end{array} & \text { scie à guichet } \\ \text { pruning saw }(25-50 \mathrm{~cm}) & \text { scie passe-partout } \\ \text { grafting saw }(25-35 \mathrm{~cm}) & \text { scie de jardinier } \\ \text { pattermaker's saw }(\mathrm{env} .19 \mathrm{~cm}) & \text { scie à greffer }\end{array}$

Dans le reste de l'Europe, cependant, les scies à main sont moins populaires (les ouvriers préfêrent encore utiliser la traditionnelle scie montée), et ce groupe de scies y est donc moins important. Ainsi, les scies à main que l'on retrouve dans les catalogues du XVIIIe siècle sont décrites comme étant soit des importations d'Angleterre, ou des États-Unis, soit des imitations des scies anglaises ou américaines.

28. CROSS-CUT HAND SAW, CROSS CUT SAW , HAND SAW , CROSSCUT HANDSAW : EGOÏNE Á TRONÇONNER, SCIE A TRONÇONNER, SCIE DE TRAVERS $_{2}$, ÉGOÏNE DE DÉBIT, ÉGOÏNE 2 .

Scie à main, de dimension moyenne, réservée au sciage droit de débit second à travers les fibres du bois.

L'égoïne à tronçonner $(56$ à $65 \mathrm{~cm}$ ) remplace les scies montées dites de travers dans les cas où leur monture est gênante. Ses dents sont pointues et biseautées : les pointes coupent le bois comme des couteaux, en laissant deux traits parallèles, tandis que 
l'intérieur des pointes cisaille le bois et le réduit en sciure ; de plus, elles sont avoyées alternativement, afin d'éviter le coincement de la lame dans le trait. L'ouvrier l'utilise pour le débit transversal de larges pièces ou de plateaux de contre-plaqué.

Remarque. "Cross-cut hand saw " est la forme étoffée ; " hand saw " est l'appellation la plus courante, quoique impropre. L'égoïne de travers est l'égoïne que l'ouvrier utilise le plus souvent et l'appellation générique " hand saw " a remplacé peu à peu celle spécifique de "crosscut hand saw"; on réserve aujourd'hui le terme " crosscut saw " à la scie passe-partout. Le fait qu'en français " égoïne " désigne de plus en plus souvent l'" égoïne de débit " s'explique de la même façon.

\section{RIP SAW, RIPSAW, HAND RIP SAW, RIPPING SAW : ÉGOÏNE À REFEN- DRE, SCIE Â REFENDRE .}

Scie à main, de dimension moyenne, réservée au sciage droit de débit second dans le sens des fibres du bois.

L'égoïne à refendre, la plus longue des scies à main $(70$ à $75 \mathrm{~cm})$, remplace les scies montées dites de long dans les cas où leur monture est gênante. Ses dents sont droites et ont la forme de petits ciseaux à bois : elles sont plates avec des tranchants parallèles. Les arêtes avant des dents coupent les fibres du bois, tandis que la face des dents réduit le bois en petits copeaux.

L'ouvrier l'utilise pour le débit longitudinal de larges pièces ou de panneaux de contre-plaqué.

30. GRAFTING SAW (G.B.), GRAFTER SAW (É.U.), GRAFTER (É.U.) (1884) SCIE Ã GREFFER.

Scie à main de petite dimension, réservée au débit à travers les fibres du bois.

La scie à greffer est une scie à main de travers, de petite dimension $(25$ à $35 \mathrm{~cm})$, à denture fine. On la retrouvait surtout en Angleterre et aux États-Unis. L'utilisation de cette scie, aujourd'hui disparue, n'est pas très claire : servait-elle à greffer, ou à enter ?

La scie à greffer peut avoir effectivement été utilisée pour différentes opérations rattachées aux greffes telles que le dégagement de branches embarrassantes, la coupe du scion à greffer, etc. Un catalogue américain de 1884 définit bien la "grafter» comme étant une petite scie à main étroite, à fine denture, destinée à l'émondage de branches en vue de l'insertion d'une greffe.

Cependant l'utilisation, pour ce genre de travail, d'une petite scie à main sans particularités semble étrange ; une scie de jardinier, en effet, aurait fait tout aussi bien l'affaire. D'ailleurs, la scie à greffer, que l'on voit dans un catalogue alsacien de 1875 , ressemble bien à une scie de jardinier : elle y est représentée comme une sorte de couteau à pain robuste, aux dents grossières (toutefois, la scie à greffer anglaise, de type égoïne, apparaît également dans ce catalogue).

Enfin, une petite scie à main est également utilisée par les ouvriers pour enter des pièces de bois, c'est-à-dire les assembler bout à bout (réparation d'un bas de porte, p. ex.) ou encore pour l'exécution d'assemblages à mi-bois.

Quoi qu'il en soit, la scie a bel et bien existé et doit donc être mentionnée...

Remarque. Il est intéressant de noter que, en anglais, "to graft " a deux acceptions : celle de " greffer " et celle d'" enter ". De même, en français, " enter " a les sens de " greffer " et d'" enter ". La dénomination "scie à enter " aurait peut-être été préférable à celle de "scie à greffer " : elle refléterait ainsi l'ambiguité contenue dans la dénomination anglaise. 


\section{PANEL SAW, PANNEL SAW, GENTLEMAN'S SAW (arch.) : SCIE À PAN- NEAU, SCIE Ä PANNEAUX.}

Scie à main, de petite dimension, réservée au sciage droit de débit second à travers les fibres du bois.

La scie à panneau est une scie à main de petite dimension $(25$ à $50 \mathrm{~cm})$, légère et à denture fine. Le menuisier l'utilise pour les sciages transversaux quand il veut une coupe plus fine et plus nette que celle que produit une égoïne de travers (minces panneaux, contre-plaqués, larges tenons, p. ex.).

Remarque. Le terme "gentleman's " apparaît dans les catalogues du XIX siècle et désigne toute une famille d'outils plus petits et plus légers que ceux réservés aux artisans ; ces outils étaient destinés aux "gentilshommes" bricoleurs. On avait ainsi, par exemple, une " gentleman's (hand) saw ", une "gentleman's (backed) saw", etc. De cette gamme d'outils, il ne reste plus aujourd'hui que la scie à main (PANEL SAW) et la scie renforcée (voir DOVETAIL SAW).

32. COMPASS SAW, LOCK SAW (G.B.), TURNING SAW (arch.), PORT SAW (ÉCOS) : SCIE À GUICHET (1694).

Scie à main, de dimension moyenne, réservée au sciage cintré de finition.

La scie à guichet est une petite égoïne (20 à $46 \mathrm{~cm}$ ) qui remplace la scie montée à chantourner dans les cas où la monture de celle-ci est gênante.

Elle est munie d'une lame épaisse, étroite et effilée, et d'une poignée ouverte de type pistolet.

Le menuisier l'utilise pour l'exécution de chantournements dits fermés, c'est-àdire au milieu d'une pièce de bois, plutôt que sur les bords de celle-ci : il perce d'abord un trou dans la pièce, puis y introduit la lame de la scie, et exécute le découpage.

33. KEYHOLE SAW, PAD-SAW, PAD SAW, FRET SAW (désuet), TURNING $\mathrm{SA}_{4}$ : PASSE-PARTOUT $_{3}$ (n.m.), SCIE BAÏONNETTE, SCIE À VOLEUR.

Scie à main, de petite dimension, réservée au sciage cintré de finition.

La scie passe-partout est une scie de menuisier semblable à la scie à guichet; elle est cependant de dimension plus petite $(15$ à $30 \mathrm{~cm}$ ), et sa lame est plus étroite.

Elle peut être munie d'une poignée ouverte, de type pistolet, ou d'une poignée droite, de métal ou de bois, dans laquelle on fixe la lame à l'aide de petites vis (d'où les termes "pad" et " baïonnette").

On l'utilise pour entamer le milieu d'une planche, pour pratiquer des ouvertures de petit diamètre, carrées ou circulaires (trous de serrure, p. ex.) qui ne peuvent être réalisées par la scie à guichet, pour exécuter de petits chantournements, ou encore pour découper des rainures.

Remarque 1. Le terme " fret saw " apparaît dans les catalogues du XIXe siècle pour désigner la petite scie passe-partout. Cependant, depuis 1914 cette appellation anglaise est réservée à la "scie de marqueterie".

Remarque 2. On qualifiait autrefois de "turning" toute scie qui servait à exécuter des sciages cintrés (voir BOW SAW et $_{3}$ OPEN PIT SAW (NARROW)). Le terme ne s'applique plus aujourd'hui qu'à la scie à monture anglaise "BOW SAW 3 ". 


\section{PRUNING SAW, GARDNER'S SAW (arch.) : SCIE DE JARDINIER, SCIE À ÉMONDER, SCIE D’ÉLAGUAGE, SCIE À ELAGUEUR.}

Scie à main, de dimension moyenne, réservée à l'émondage.

La scie de jardinier est une scie à main de travers de dimension moyenne (25 à 50 $\mathrm{cm}$ ). Elle existe sous plusieurs formes; ainsi, elle peut parfois ressembler à une petite égoïne de travers, ou encore être munie de dents sur les deux rives de la lame, etc., mais le plus souvent sa lame est courbe, en forme de serpe, l'arête concave étant seule munie de dents. De plus, la poignée peut être ouverte, fermée, ou encore creuse, pour permettre de fixer la lame au bout d'un long manche. Le jardinier l'utilise pour émonder les arbres.

\section{FLOORING SAW, INSIDE START SAW (É.U.) (rare) : SCIE DÉFONCEUSE.}

Scie à main, de petite dimension, réservée au sectionnement de lames de parquets.

La scie défonceuse est une scie à main particulière, de petite dimension (30 à 36 $\mathrm{cm}$ ) : les deux rives de la lame sont munies de dents. De plus, le bout de la lame est pointu et le dos, qui est convexe à cet endroit, permet à l'ouvrier de commencer le trait de scie au milieu d'une pièce de bois.

On utilise la scie défonceuse pour sectionner des lames de parquets, ayant déjà été posées, sans endommager les lames avoisinantes (lors de réparations de plomberie, $\mathrm{p}$. ex.).
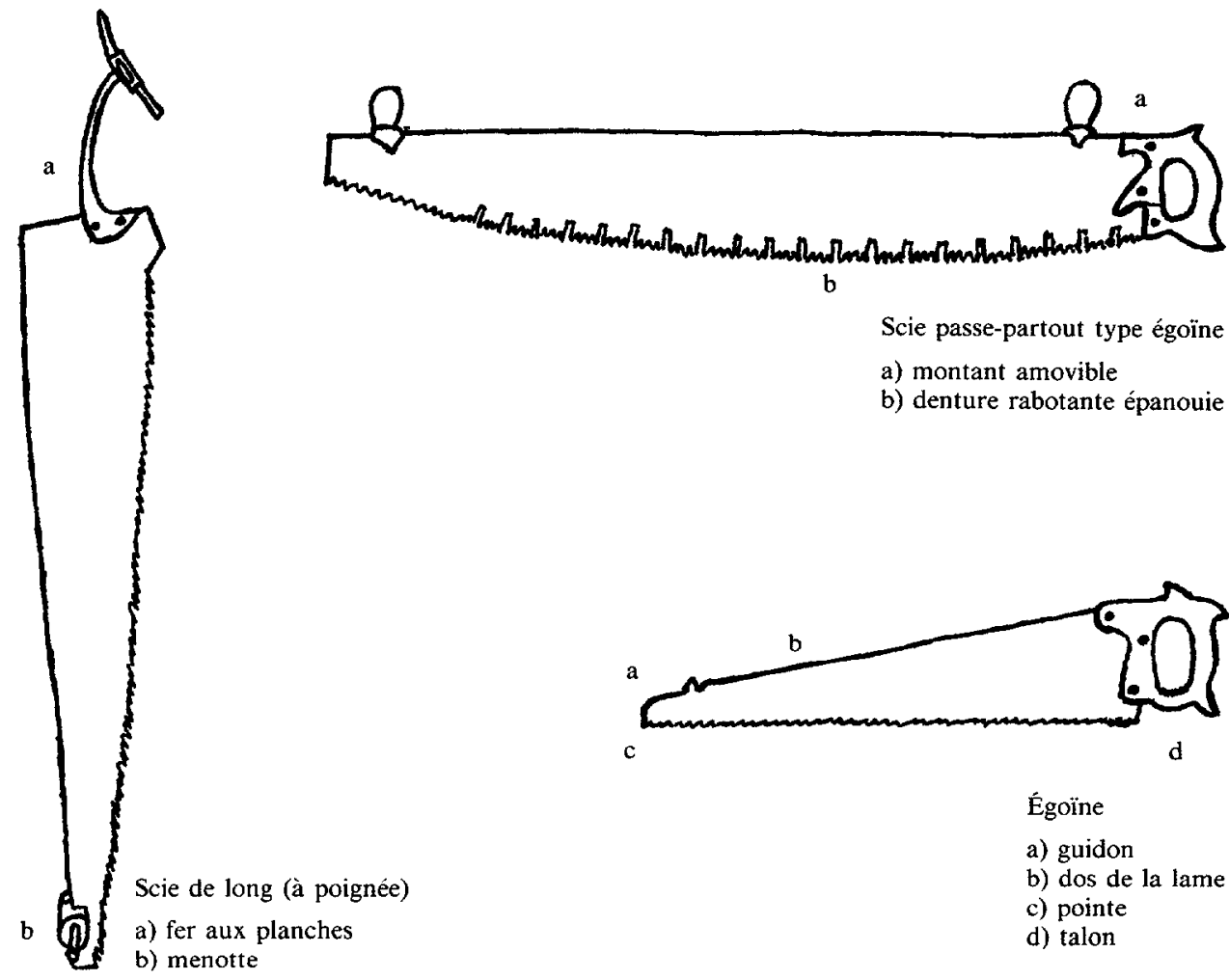

a) guidon

b) dos de la lame

c) pointe

b) menotte

d) talon 


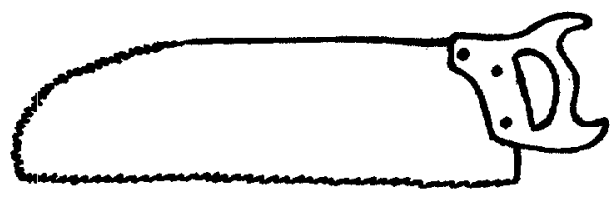

Scie défonceuse
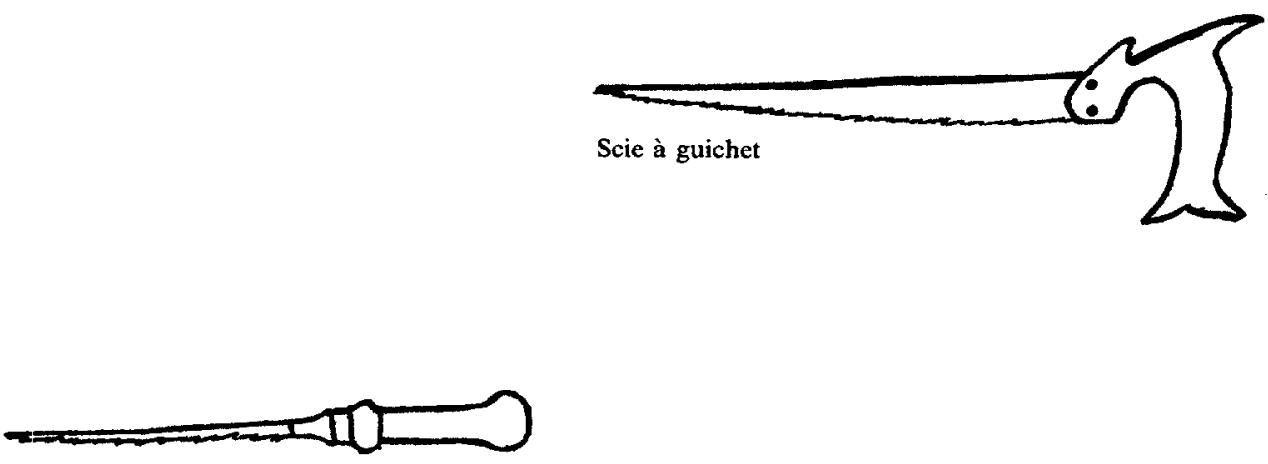

Scie baïonnette

Marie ProulX 